\title{
HYPERCHAOS FROM A MODEL OF COUPLED STRATOSPHERE-TROPOSPHERE DYNAMICS
}

\author{
SUSHEEL ADUSUMILLI \\ Scripps Institution of Oceanography, University of California at San Diego, 9500 Gilman Drive, La Jolla, \\ CA 92093 USA \\ suadusum@ucsd.edu \\ ROBERT A. VAN GORDER \\ Mathematical Institute, University of Oxford \\ Andrew Wiles Building, Radcliffe Observatory Quarter, Woodstock Road \\ Oxford OX2 6GG United Kingdom \\ Robert.VanGorder@maths.ox.ac.uk
}

Received (to be inserted by publisher)

\begin{abstract}
We present a six-dimensional system describing coupled troposphere-stratosphere dynamics which takes the form of two coupled Lorenz-84 systems (one for each of the troposphere and stratosphere) involving thermal forcing terms. The systems are coupled through a linear interaction term, which permits energy transfer between both troposphere and stratosphere layers. While other six-dimensional systems giving hyperchaos and multi-scroll attractors have been found in the literature, the coupled systems given here arises naturally from the physical problem. In particular, the resulting six-dimensional system constitutes a physically interesting model where the stratosphere-troposphere dynamics are coupled to one another (rather than just coupling the troposphere dynamics to the stratosphere, while keeping the time evolution of the stratosphere independent). This model gives bounded dynamics and for some parameters exhibits chaos or hyperchaos. Interestingly, there are parameter regimes for which the dynamics go directly between periodic orbits and hyperchaos, bypassing an intermediate chaos step. The precise form of the coupling between the two Lorenz-84 systems is found to strongly influence the solution behavior. We find that even small coupling from the stratosphere back to the troposphere can destabilize the system and yield hyperchaotic dynamics, while for other parameter sets this coupling can instead smooth dynamics in both regions.
\end{abstract}

Keywords: stratosphere-troposphere dynamics; hyperchaos; Lyapunov exponents; chaotic attractors; generalized coupled Lorenz system

\section{Introduction}

The first model giving the so-called hyperchaos was due to Rössler [1979]. In order to observe hyperchaos, a system must have bounded trajectories in phase space, yet two Lyapunov exponents must remain positive. A necessary condition for a continuous-time dynamical system to be hyperchaotic is therefore that the dimension of the system is at least four. As a result, a number of three-dimensional systems known to give chaos (bounded trajectories with one positive Lyapunov exponent) have been extended, through the addition of extra coupling terms and equations, to obtain higher-dimensional systems exhibiting hyperchaos 
[Baire and Sahle, 1995; Baire and Thomsen, 1993; Choudhury and Van Gorder, 2012; Gao et al., 2006; Nik and Van Gorder, 2013]. Many of these models are therefore mathematical constructs developed to give a certain behavior in their solutions. However, there has been work in development of physically meaningful models which happen to give hyperchaos, with applications being found in neuronal networks [Shuai et al., 1997], optics [Udaltsov et al., 2001], semiconductors [Peinke et al., 1993], communications [Xiao et al., 1996], nonlinear antiferromagnetic resonance [Moser eta al., 1993], intermittent convection in a magnetized fluid [Macek and Strumik, 2014], and networks [Kestler et al., 2007]. Work has also been taken in order to control [Colet et al., 1994; Kapitaniak, 1994; Huang, 2004] or synchronize [Ali and Fang, 1997; Bryant, 2010; Peng et al., 1996; Tamasevicius and Cenys, 1997] the emergent hyperchaos in such models. Since hyperchaos is observed when two Lyapunov exponents become positive, one can be interested in finding hyperchaos in parameter regimes near where chaos is already known to exist, and this is related to the study of the transition between chaos and hyperchaos in dynamical systems [Kipitaniak, 1993; Kipitaniak et al., 2000; McCullen and Moresco, 2011; Pavlov et al., 2015].

It was originally the study of atmospheric dynamics that would lead Lorenz to obtain a dynamical system giving chaos [Lorenz, 1963], and in this sense there was a natural motivation for the study of chaos. As mentioned above, many existing hyperchaotic systems are constructed to mathematically exhibit hyperchaos. However, we shall present a model - fittingly motivated by atmospheric dynamics - which exhibits hyperchaos in a fairly natural way. The model constitutes a physically interesting scenario when the troposphere-stratosphere dynamics are coupled to one another, rather than just a coupling of the stratospheric dynamics to the troposphere by assuming the stratospheric dynamics take a prescribed, simple form. While the system may have zero, one, or two positive Lyapunov exponents, we note that the bifurcation diagrams suggest that is it possible for the system to go directly between zero and two Lyapunov exponents. This corresponds to a switch between periodic orbits and hyperchaos, bypassing any intermediate standard (one positive Lyapunov exponent) chaos. The primary bifurcation parameters will be due to the coupling of the two layers, as well as constant (averaged) forcing terms due to topography. We start with a review of the relevant atmospheric dynamics, after which we introduce the six-dimensional dynamical system governing the coupled troposphere-stratosphere interaction. We demonstrate that the dynamics of the system can give periodic orbits, chaos, and hyperchaos, and bifurcation diagrams inform us of where in parameter space the coupling and forcing parameters permit each of these behaviors. We conclude by giving some physical intuition for the results.

In Sec. 2 we give a brief primer on troposphere-stratosphere interactions We also discuss the Lorenz-84 model for tropospheric dynamics. Motivated by this model, we consider a coupled Lorenz- 84 model which has an extra three-dimensional component for the stratospheric dynamics in Sec. 3. This allows for each of the respective troposphere and stratosphere dynamics to influence the other. We study the possible dynamics of this model as a function of the strength of asymmetric forcing terms and parameters coupling tropospheric and stratospheric dynamics. In Sec 4, we apply a competitive modes analysis in order to obtain more general parameter families which may lead to chaos in our model. We summarize and relate our back to atmospheric turbulence near the tropopause in Sec. 5.

\section{Stratosphere-Troposphere Dynamics}

Conventionally, the atmosphere is divided into various layers, the lowest of which is the troposphere [Matthewman et al., 2009]. The troposphere extends to $8-15 \mathrm{~km}$ above the surface, and is separated from the next layer, the stratosphere, by the tropopause. The stratosphere is characterized by a reversal of the temperature gradient, caused by the presence of ozone and the absorption of solar UV radiation. The majority of current weather forecasts focus on the dynamics of the troposphere, which varies erratically, limiting any predictive skill to the order of a few days. The climate in the stratosphere is much less erratic. However, until recently, the effect of the stratosphere on tropospheric dynamics had been thought to be minimal. In the northern hemisphere winter (December-January-February), the stratosphere is subject to greater heating in lower latitudes, which drives the mid-latitude westerly winds - this creates a vortex, called the stratospheric polar vortex. It is the effect of this stratospheric polar vortex on the winter climate in the troposphere that motivates our current investigation. 


\section{X - System}

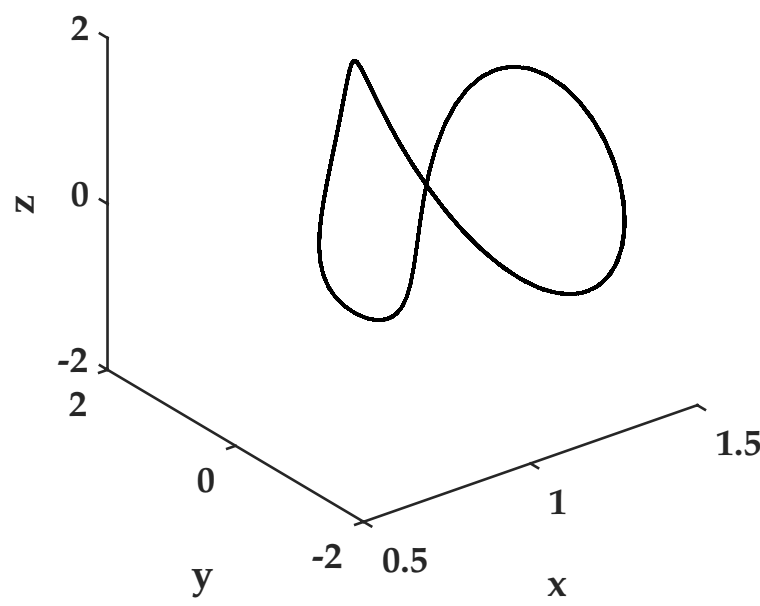

Y - System

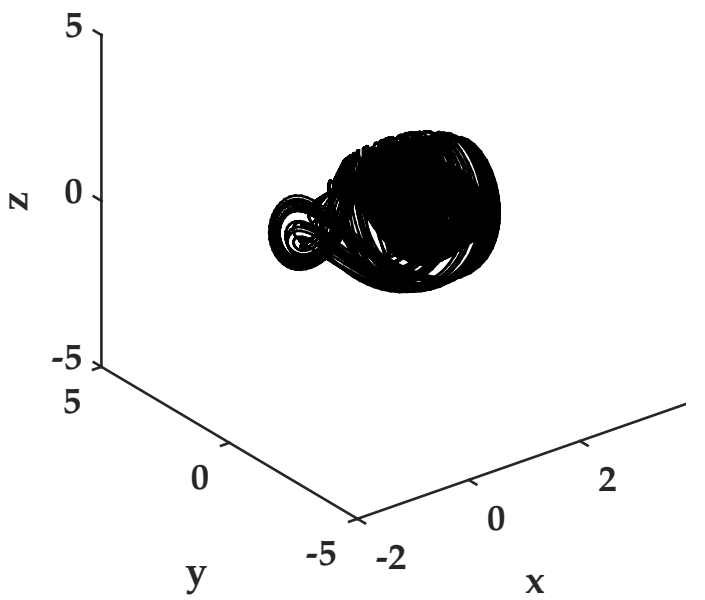

Fig. 1. Phase portrait of the tropospheric Lorenz-1984 model, given by (1). The parameters used here are $a=0.25, b=4$, $F=8$ and $G=0.5$ for the X system and $G=1$ for the Y system. The initial conditions were chosen so that they were close to the attractor.

Heights above mean sea level are usually weighted by the gravity at the elevation and latitude. Geopotential height is often referred to by the average pressure at the location, and a geopotential anomaly is the variation from that average at various times. The geopotential anomaly is measured by several reanalysis projects [Dee et al., 2011; Kistler et al., 2001]. In terms of the predictability of the northern hemisphere weather, one of the particularly relevant meteorological variables is the pressure anomaly (deviations from the average pressure) in the lower troposphere (measured here using data from $1000 \mathrm{hPa}$ geopotential height anomalies). The hemispheric mode of height/pressure variability is related closely to the Arctic Oscillation [Thompson et al., 2000]. Another similar measure of northern hemisphere pressure variability is the North Atlantic Oscillation, and this is defined as the difference in pressure levels between Iceland (at Stykkisholmur or Reykjavik) and Portugal (at Azores or Lisbon) [Solomon, 2007]. Measures similar to the Arctic Oscillation can be made for all levels in the atmosphere (not just the troposphere), and we call the collection the Northern Annular Mode (NAM).

The Lorenz-84 system [Lorenz, 1984], which was designed to broadly characterize the atmospheric irregularity that is inherent in the northern hemisphere, can be viewed as a prototypical model of the NAM. The Lorenz- 84 model is given by the equations

$$
\begin{aligned}
& \dot{x}=-y^{2}-z^{2}-a x+a F, \\
& \dot{y}=x y-b x z-y+G, \\
& \dot{z}=b x y+x z-z .
\end{aligned}
$$

Motivation for this model is described in detail in Lorenz [1984]. Here, $x$ is a time-dependent variable that represents the westerly winds, a major component of the polar vortex. The variables $y$ and $z$ represent eddies that can transfer heat and momentum to the higher latitudes. These eddies can be amplified when they interact with the westerlies, and these interactions are incorporated into the $x y$ and $x z$ terms. Correspondingly, at the same time, the westerlies would then lose energy, and this is accomplished through the $-y^{2}$ and $-x^{2}$ terms. Other $x$ forcing terms include $a F$, which is the symmetric thermal forcing given in this case by the annual solar cycle. In the model, we also have $G$, which is its asymmetric counterpart (for instance, this could be due to the orography). The natural dampening of all the quantities involved is given by the linear terms. Multiplying (1) by $x, y$ and $z$ respectively, it is straightforward to see that

$$
\frac{1}{2} \frac{d}{d t}\left(x^{2}+y^{2}+z^{2}\right)=-a x^{2}-y^{2}-z^{2}+a F x+G y .
$$


Since $a, F$, and $G$ are positive in this model, it can be seen that the total energy in the system will decrease if the state variables exceeds certain value, hence the system is bounded. The phase portrait of this system is shown in Fig. 1. It can be seen that the system spends most of its time in one of two states - this is interpreted in meteorological terms as two climate regimes. Such behavior, when it persists, is called intransitivity [Lorenz, 1990]. Since the publication of the original model, several modifications were proposed to it, including a modification by Lorenz [1990] that added a periodic term for $F$ to simulate the annual solar cycle.

\section{Coupled Lorenz-84 System for Troposphere-Stratosphere Interactions}

In the analysis of coupled stratosphere-troposphere interactions, we propose a coupled Lorenz-84 system as a proxy for the atmospheric dynamics. One component, $X$, is (very crudely) representative of the troposphere and shows steady recurrent dynamics [Adusumilli, 2015], while the other $(Y)$ represents the stratosphere. This system is given by the equations

$$
\begin{aligned}
& \dot{x}_{X}=-y_{X}^{2}-z_{X}^{2}-a x_{X}+a F, \\
& \dot{y}_{X}=x_{X} y_{X}-b x_{X} z_{X}-y_{X}+G_{X}+\epsilon_{X}\left(y_{X}-y_{Y}\right), \\
& \dot{z}_{X}=b x_{X} y_{X}+x_{X} z_{X}-z_{X}, \\
& \dot{x}_{Y}=-y_{Y}^{2}-z_{Y}^{2}-a x_{Y}+a F, \\
& \dot{y}_{Y}=x_{Y} y_{Y}-b x_{Y} z_{Y}-y_{Y}+G_{Y}+\epsilon_{Y}\left(y_{Y}-y_{X}\right), \\
& \dot{z}_{Y}=b x_{Y} y_{Y}+x_{Y} z_{Y}-z_{Y} .
\end{aligned}
$$

In the basic stratosphere-troposphere model considered here, we use a lower value of $G_{Y}$ for the stratosphere than we take for the value of $G_{X}$ in the troposphere, since the asymmetric forcing (that can have a significant impact on the troposphere due to the orography, for instance) might be restricted in the less chaotic stratosphere Here $\epsilon_{X}$ gives the coupling of stratospheric dynamics into the troposphere, while $\epsilon_{Y}$ gives the coupling of tropospheric dynamics into the stratosphere. While the idea of the stratosphere having an influence on the upper troposphere is not new, we note that there has also been some speculation on the influence of the upper troposphere on the stratosphere, as well [Haynes et al., 1995]. Therefore, we include both manners of coupling.

Note that this coupled Lorenz 84 model is fundamentally different in both structure and physics from the coupled Lorenz systems of Ma et al. [1997] and Grassi et al. [2009]. In both of those papers, the coupled Lorenz systems described were constructed to study chaos, hyperchaos, multiple scroll attractors, and other dynamics, yet they were primarily mathematically motivated. The coupled Lorenz system we study is primarily motivated by the physical application, and it is interesting to see a physically interesting (rather than mathematically contrived) example of a system such as this giving hyperchaos.

As we did for the uncoupled model (1), we can show for (3) that

$$
\begin{aligned}
\frac{1}{2} \frac{d}{d t} & \left(x_{X}^{2}+y_{X}^{2}+z_{X}^{2}+x_{Y}^{2}+y_{Y}^{2}+z_{Y}^{2}\right) \\
= & -a\left(x_{X}^{2}+x_{Y}^{2}\right)-\left(z_{X}^{2}+z_{Y}^{2}\right)+a F\left(x_{X}+x_{Y}\right) \\
& -\left(1-\epsilon_{X}\right) y_{X}^{2}-\left(1-\epsilon_{Y}\right) y_{Y}^{2} \\
& -\left(\epsilon_{X}+\epsilon_{Y}\right) y_{X} y_{Y}+G_{X} y_{X}+G_{Y} y_{Y} .
\end{aligned}
$$

While (2) implies boundedness of trajectories of solutions to (1), note that (4) implies that trajectories of solutions to (3) are bounded conditionally, depending on $\epsilon_{X}$ and $\epsilon_{Y}$. We find that a sufficient condition for trajectories of solutions to (3) to be bounded is the parameter restriction $4\left(1-\epsilon_{X}\right)\left(1-\epsilon_{Y}\right)>\left(\epsilon_{X}+\epsilon_{Y}\right)^{2}$ where $0<\epsilon_{X}, \epsilon_{Y}<1$. This defines a convex subset of the region $0<\epsilon_{X}, \epsilon_{Y}<1$. However, we should note that we have found numerical solutions which remain bounded even outside of this region. Hence, this region defines a sufficient but not necessary condition for boundedness of trajectories to (3).

The phase portraits for the two components with $G_{X}=0.5$ for the troposphere and $G_{Y}=0.1$ for the stratosphere are shown in Fig. 2. Here, we use a coupling strength of $\epsilon_{X}=4$ (which measures the degree to which the stratosphere dynamics influence the troposphere dynamics). It shall usually make sense to 

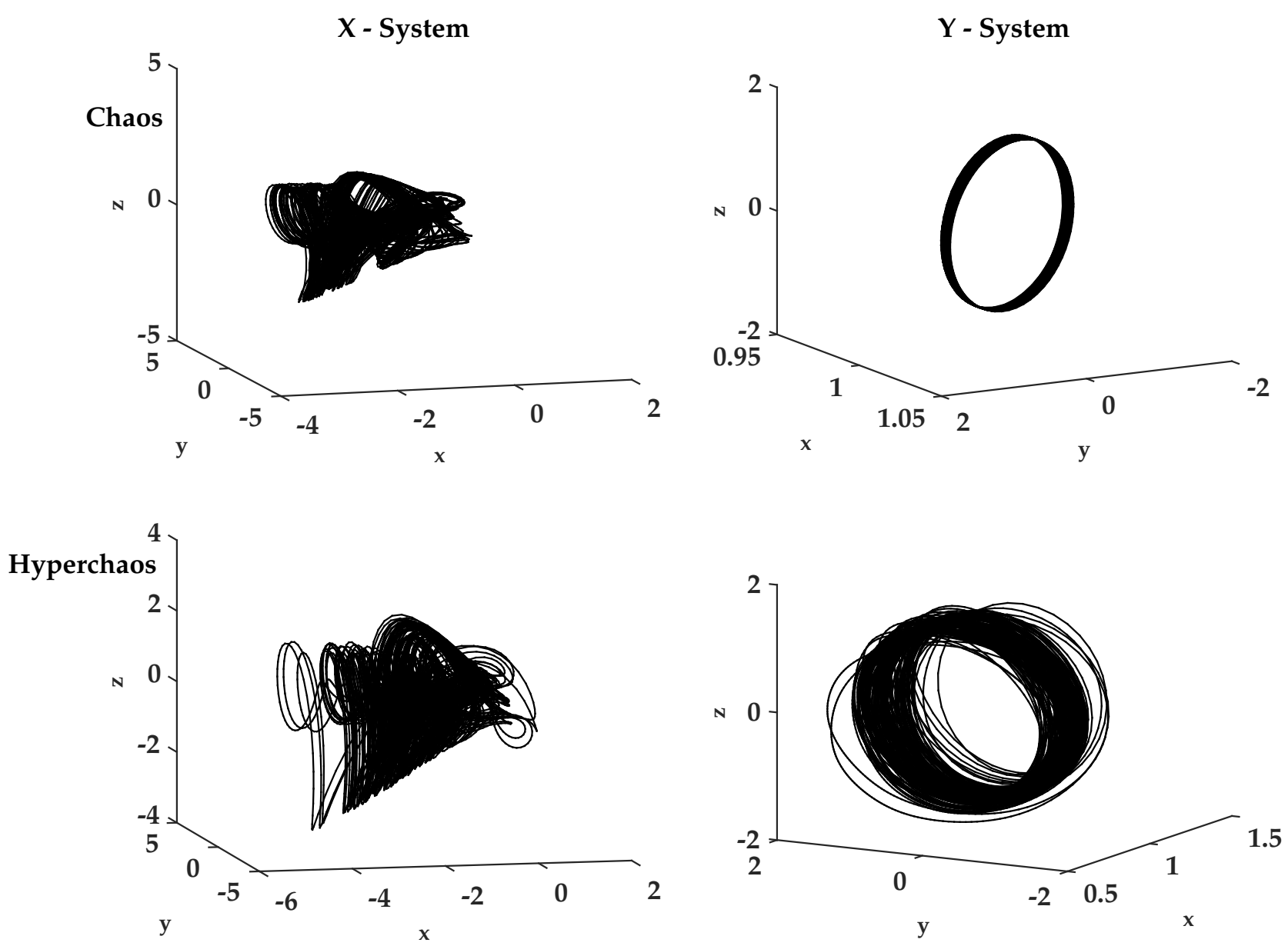

Fig. 2. Transition from chaos to hyperchaos for the coupled L84 system with the tropospheric component on the left column and the stratospheric component on the right column. The parameters used here are $a=0.25, b=4, F=8, G_{Y}=0.1$, $G_{X}=0.5$ with $\epsilon_{X}=4, \epsilon_{Y}=0$ (for chaotic dynamics) or with $\epsilon_{X}=4, \epsilon_{Y}=0.25$ (for hyperchaotic dynamics). Even for small asymmetric forcing and small coupling from the troposphere to the stratosphere, we see the emergence of chaos in the stratospheric part of the model, which drives the entire system (3) into hyperchaos.

consider $\epsilon_{X}>\epsilon_{Y}$, since the influence of the troposphere on the stratosphere is less pronounced. When we activate the parameter $\epsilon_{Y}$, we have a feedback effect since the troposphere dynamics will then influence the stratosphere. As we see from Fig. 2, this pushes the observed dynamics from chaos to hyperchaos. As seen in the lower panels of Fig. 2, both component systems are chaotic, and we can verify that the resulting six-dimensional dynamical system has two positive Lyapunov exponents for the parameter values taken. To see this influence of the coupling parameters $\epsilon_{X}$ and $\epsilon_{Y}$ on these dynamics more generally, we obtain a bifurcation diagram by evaluating the Lyapunov exponent spectrum of this system using the Wolf algorithm [Wolf et al., 1985]. In Fig. 3 we plot a bifurcation diagram which shows the number of positive Lyapunov exponents of the bounded trajectories of (3) as a function of the coupling parameters, $\epsilon_{X}$ and $\epsilon_{Y}$. We label the dynamics for each based on what we observe in the corresponding phase portraits. We see that for the fixed choice of parameters taken, there is a large band of chaotic dynamics which surrounds an inner band of hyperchaotic dynamics. While the values of $\epsilon_{X}$ or $\epsilon_{Y}$ needed for chaos appears to be large, note that this is due to the values of the other parameters. For other parameter choices, one obtains chaos for even small values of $\epsilon_{X}$ and $\epsilon_{Y}$. The bifurcation diagram in Fig. 3 is chosen to show a case where hyperchaotic dynamics are surrounded by chaotic dynamics in parameter space. As we shall see later, this is not always the case.

The thermal forcing parameters will also strongly influence the dynamics of the coupled system (4). In Fig. 4 we provide bifurcation diagrams in the thermal forcing parameters for each of the troposphere 


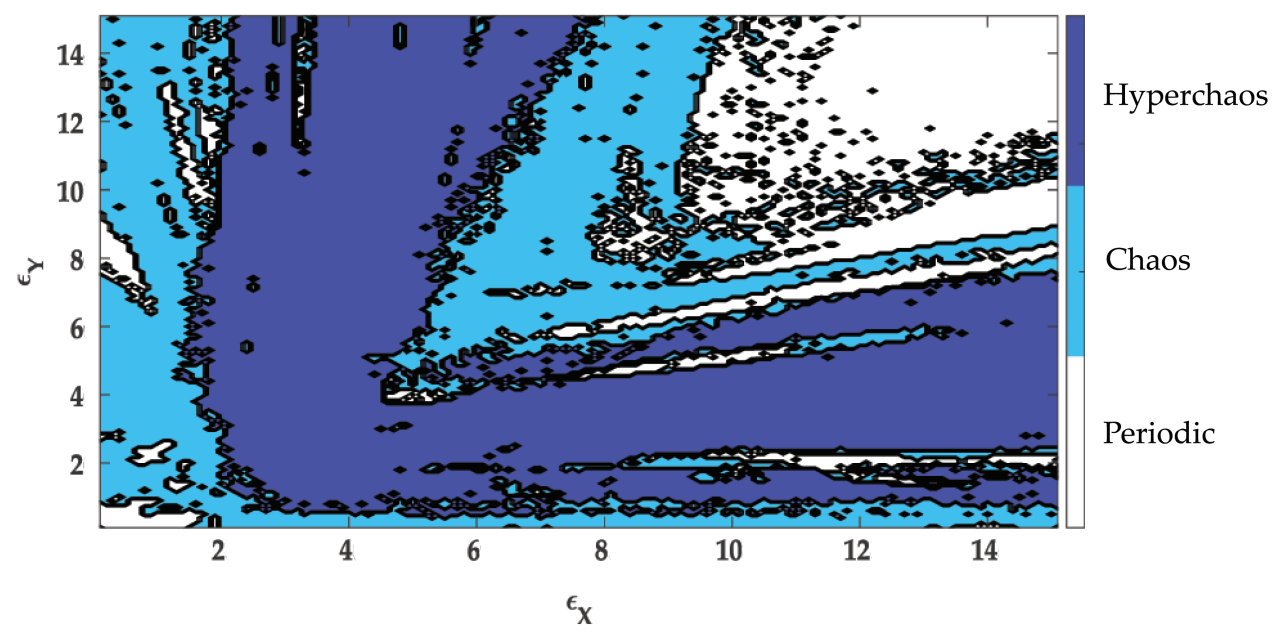

Fig. 3. Bifurcation diagrams in coupling parameters $\epsilon_{X}$ and $\epsilon_{Y}$ showing parameter regions giving periodic orbits, chaos, and hyperchaos. The parameters used here are $a=0.25, b=4, F=8, G_{Y}=0.1$ and $G_{X}=0.5$. Even for larger values of the coupling parameters $\epsilon_{X}$ and $\epsilon_{Y}$ we find bounded trajectories which lead to chaos or hyperchaos.

and stratosphere, $G_{X}$ and $G_{Y}$, respectively. While both parameters play a role in the dynamics, we see that it is the thermal forcing in the troposphere that more readily changes the qualitative features of the solutions, with the bifurcation diagrams exhibiting a banded structure due to an increase in $G_{X}$. Note that hyperchaos is possible even with $\epsilon_{Y}=0$, provided that the forcing term $G_{X}$ is large enough. On the other hand, when $\epsilon_{Y}$ is increases, the threshold value of $G_{X}$ which allows for hyperchaos lowers, meaning that it is easier to observe hyperchaos when there is a stronger coupling of the stratosphere to the troposphere dynamics.

While a stronger coupling of the stratosphere and troposphere permits hyperchaos for smaller thermal forcing parameter $G_{X}$, note also that there may be a smoothing effect for fixed $G_{X}$ when we increase the coupling between the stratosphere and troposphere components. This is best seen by comparing the top and bottom diagrams in Fig. 4 . For, say, $G_{Y}=0.1$ and $G_{X}=1$, the system with $\epsilon_{X}=4$ and $\epsilon_{Y}=0$ gives hyperchaos, while if we increase the stratospheric coupling term to $\epsilon_{Y}=0.25$, we obtain regular (non-chaotic) periodic dynamics for large time. Therefore, one must be mindful of all parameter values, as in some parameter regimes they may conspire to give hyperchaos, while in other parameter regimes one might observe stable regular dynamics.

One interesting feature of the system (4) is that we observe an immediate transition between regular periodic dynamics and hyperchaos. This is observed in both panels of Fig. 4 where regions in parameter space admitting hyperchaotic dynamics (dark blue regions) are adjacent to regions in parameter space admitting periodic orbits (white regions). We similarly find small regions of periodic dynamics adjacent to hyperchaotic regions, in Fig. 3. Therefore, a slight perturbation to the model parameters can push the solutions from stable periodic trajectories into the hyperchaotic regime, highlighting the sensitivity of equation (4) to the coupling and thermal forcing parameters. Furthermore, we take the view that the hyperchaos generated here is rather fundamental, due to the fact is is easy to move into a hyperchaotic regime. Indeed, rather than being a small subset of the chaotic parameter regime valid when parameters take non-physical values, hyperchaotic trajectories can be obtained even from slight perturbations of stable periodic trajectories. One such transition between periodic behavior and hyperchaos is shown in Fig. 5. Here we treat $\epsilon_{Y}$ as the bifurcation parameter; when $\epsilon_{Y}=0$, we observe hyperchaos, while when $\epsilon_{Y}=0.25$, the dynamics smooth and periodic orbits are found. Therefore, in this instance, we observe that the stronger coupling with the stratosphere actually stabilizes the entire system (4). This is keeping with the view that the stratosphere itself is primarily dissipative, with perturbations collapsing to steady state dynamics in the absence of dynamic forcing [Shepherd, 2002]. Of course, in the presence of such forcing, the dynamics can become more complicated, and for even larger values of forcing (see Fig. 4) the dynamics can be pushed back into the chaotic or even hyperchaotic regimes. 

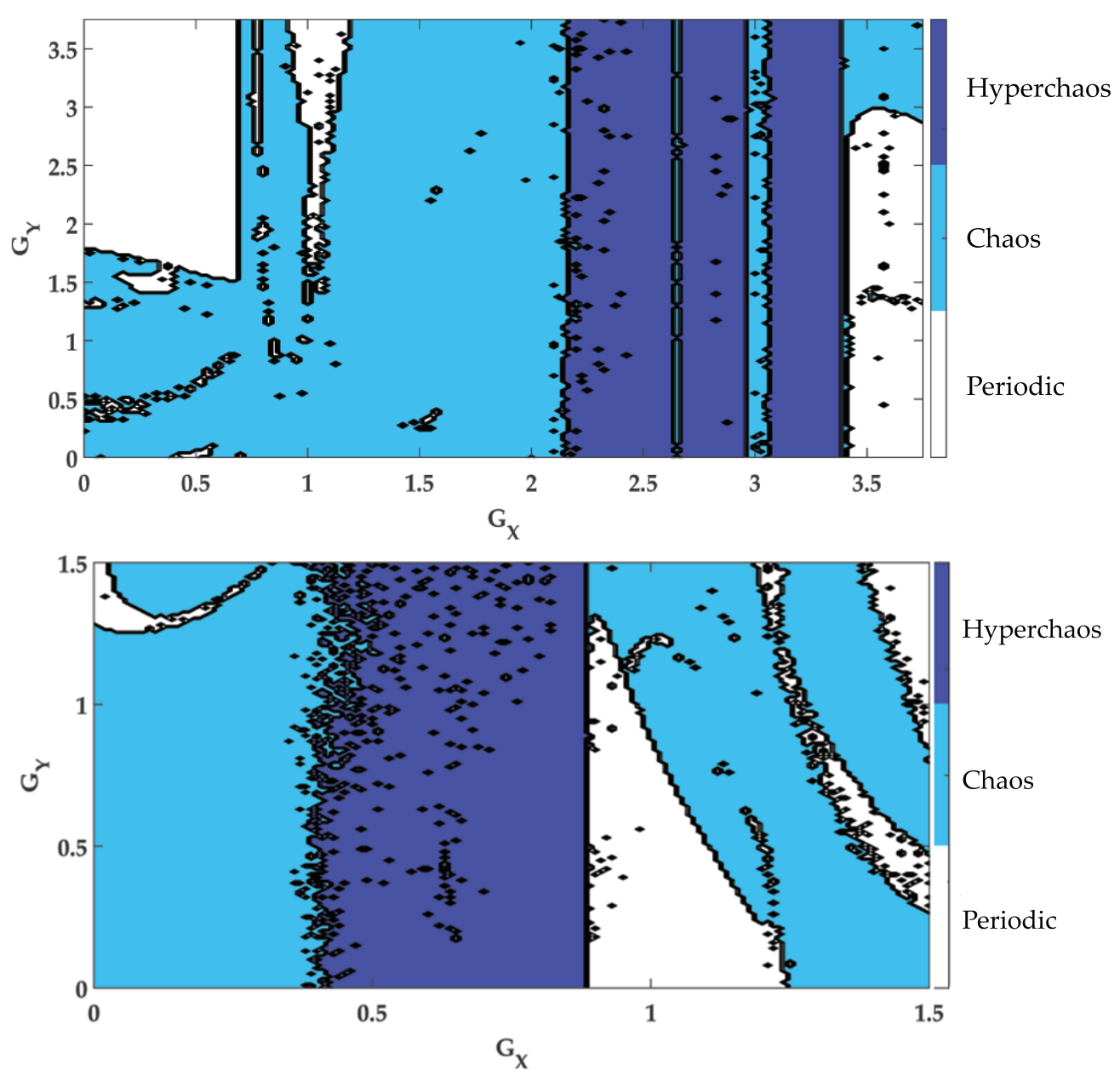

Fig. 4. Bifurcation diagrams in asymmetric thermal forcing parameters $G_{X}$ and $G_{Y}$ showing parameter regions giving periodic orbits, chaos, and hyperchaos. The parameters used here are $a=0.25, b=4, F=8, \epsilon_{X}=4$ and $\epsilon_{Y}=0$ (top diagram), $\epsilon_{Y}=0.25$ (bottom diagram) taken here to represent higher coupling from the troposphere to the stratosphere. By coupling tropospheric dynamics back into the stratosphere, even by a small amount, the threshold in $G_{X}$ needed for hyperchaos in system (3) greatly decreases. However, this behavior is not always de-stabilizing. Indeed, we note that some regions giving hyperchaos in the absence of the coupling parameter $\epsilon_{Y}$ now give regular, periodic trajectories. This highlights the relative complexity of the model.

\section{Competitive modes analysis}

In order to further study the appearance of chaotic dynamics in the coupled Lorenz- 84 systems, we shall turn our attention to a competitive modes analysis. The method of competitive modes involves recasting a dynamical system as a coupled system of oscillators [Reeves et al., 2012; Van Gorder, 2011; Van Gorder, 2013; Van Gorder and Choudhury, 2010; Yao et al., 2002; Yu, 2006; Yu et al., 2007]. For instance, consider the general nonlinear autonomous system of dimension $n$ given by

$$
\dot{\xi}_{i}=f_{i}\left(\xi_{1}, \xi_{2}, \ldots, \xi_{n}\right) .
$$

Differentiation of (5) once gives a coupled system of second order equations,

$$
\begin{aligned}
\ddot{\xi}_{i}=\sum_{j=1}^{n} f_{j} \frac{\partial f_{i}}{\partial \xi_{j}}= & -\xi_{i} g_{i}\left(\xi_{1}, \xi_{2}, \ldots, \xi_{i}, \ldots, \xi_{n}\right) \\
& +h_{i}\left(\xi_{1}, \xi_{2}, \ldots, \xi_{i-1}, \xi_{i+1}, \ldots, \xi_{n}\right) .
\end{aligned}
$$

When a $g_{i}$ is positive, its respective $i$ th equation behaves like an oscillator. The following conjecture is posed in Yao et al. [2002]: 

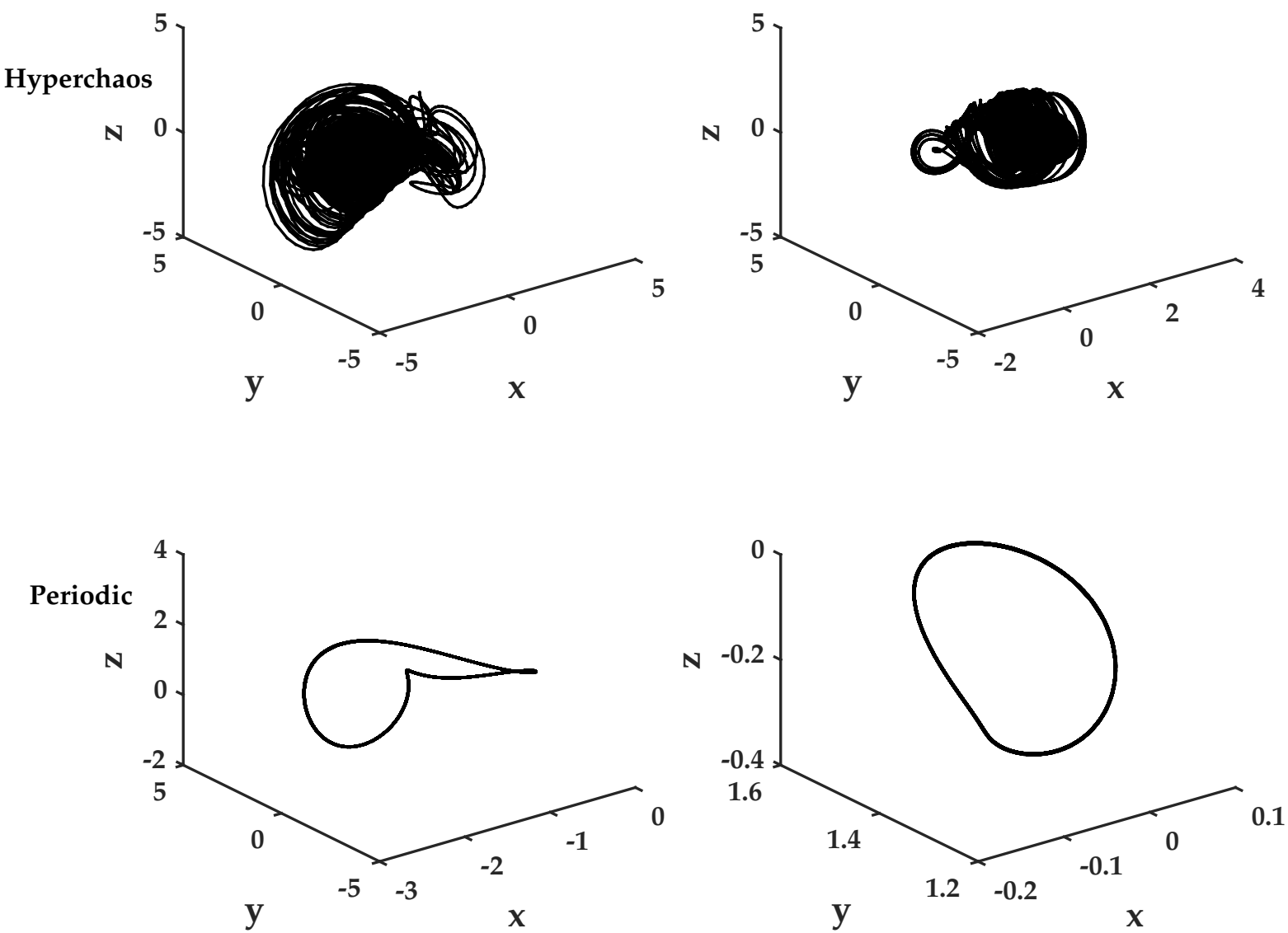

Fig. 5. Transition from hyperchaos to periodic behaviour for the coupled L84 system with the tropospheric component on the left column and the stratospheric component on the right column. The parameters used here are $a=0.25, b=4, F=8$, $G_{Y}=0.1, G_{X}=1$ with $\epsilon_{X}=4, \epsilon_{Y}=0$ (for hyperchaotic dynamics) or with $\epsilon_{X}=4, \epsilon_{Y}=0.25$ (for periodic dynamics). Instead of becoming less regular, this particular coupling between stratosphere and troposphere has actually had a stabilizing effect on the solution trajectories for (3). Therefore, additional coupling of the troposphere back into the stratosphere via $\epsilon_{Y}$ can either stabilize or destabilize the dynamics of (3), depending on the parameter regime we consider.

Competitive Modes Requirements: The conditions for dynamical systems to be chaotic are given by:

(A) there exist at least two non-zero modes, labeled $g_{i}$ in the system;

(B) at least two $g$ 's are competitive or nearly competitive, that is, for some $i$ and $j, g_{i} \approx g_{j}>0$ at some $t$;

(C) at least one of the $g$ 's is a function of evolution variables such as $t$ (this can be through the state variables $\left.\xi_{i}\right)$; and

(D) at least one of the $h$ 's is a function of state variables $\xi_{i}$.

The requirements (A)-(D) essentially tell us that a condition for chaos is that two or more equations in (6) behave as oscillators $\left(g_{i}>0\right)$, and that two of these oscillators lock frequencies at one or more times. In practice, we find that the frequencies agree at a countably infinite collection of time values [Van Gorder, 2011; Yu, 2006]. The frequencies should be functions of time (i.e., we have nonlinear frequencies), and there should be at least one forcing function which depends on a state variable. Furthermore, it has been seen that the modes should be competitive in some intermittent manner, without perfect repetition or periodicity [Choudhury and Van Gorder, 2012].

In order to apply the competitive modes analysis to our system of interest, we rewrite the system (3) 


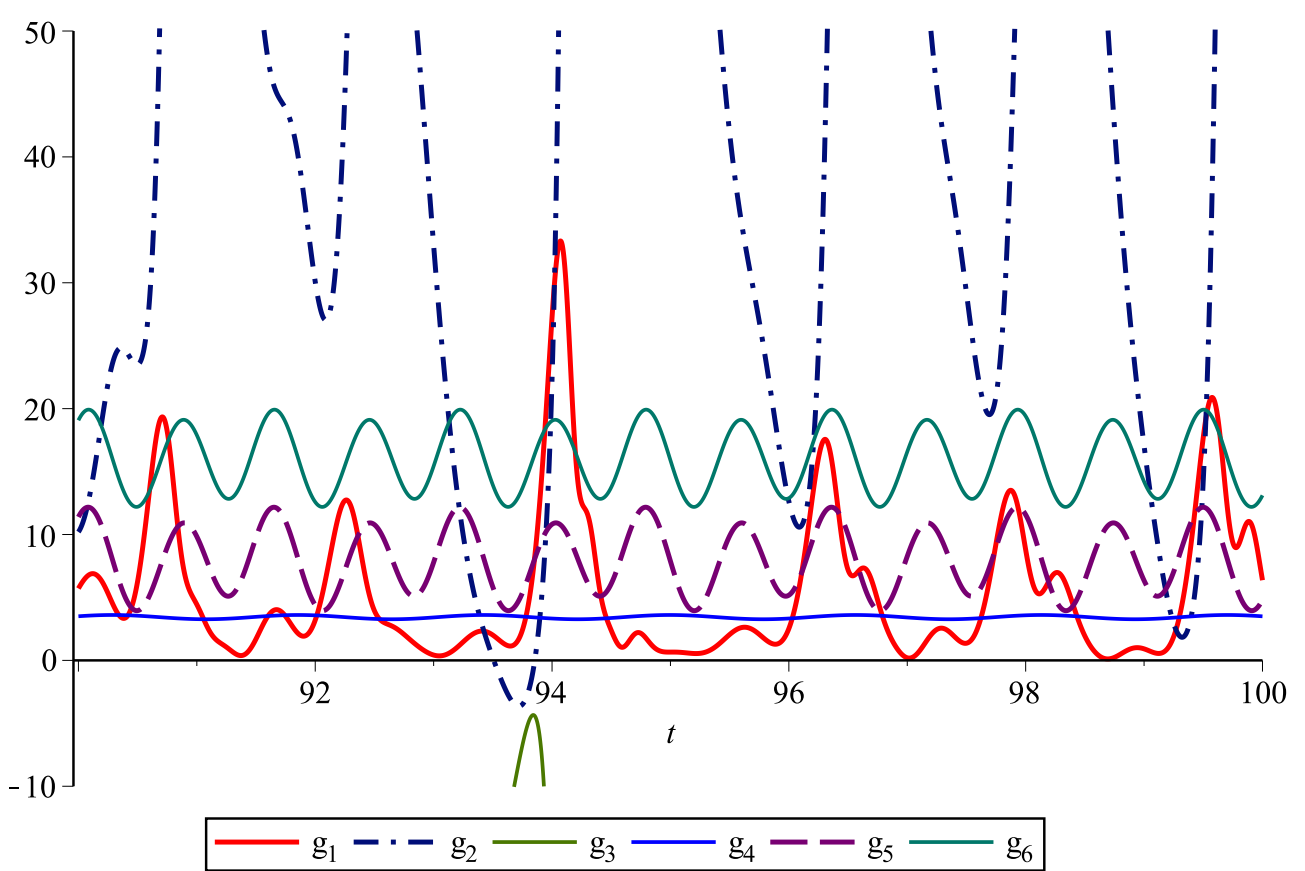

Fig. 6. Plot of the mode frequencies for the parameter values $a=0.25, b=4, F=8, G_{Y}=0.1, G_{X}=0.5, \epsilon_{X}=4, \epsilon_{Y}=0$ giving chaos.

as the system of coupled oscillators

$$
\begin{aligned}
\ddot{x}_{X}+g_{1} x_{X} & =h_{1}, \\
\ddot{y}_{X}+g_{2} y_{X} & =h_{2}, \\
\ddot{z}_{X}+g_{3} z_{X} & =h_{3}, \\
\ddot{x}_{Y}+g_{4} x_{Y} & =h_{4}, \\
\ddot{y}_{Y}+g_{5} y_{Y} & =h_{5}, \\
\ddot{z}_{Y}+g_{6} z_{Y} & =h_{6},
\end{aligned}
$$

where

$$
\begin{aligned}
& g_{1}=2 y_{X}^{2}+2 z_{X}^{2}-a^{2} x_{X}, \\
& g_{2}=y_{X}^{2}+z_{X}^{2}+\left(b^{2}-1\right) x_{X}^{2}+b y_{X} z_{X}-\epsilon_{X} \epsilon_{Y} y_{X}+\left(a+2\left(1-\epsilon_{X}\right)\right) x_{X}-a F-\left(1-\epsilon_{X}\right)^{2}, \\
& g_{3}=y_{X}^{2}+z_{X}^{2}-x_{X}^{2}+b y_{X} z_{X}+\left(b^{2}+a+2\right) x_{X}-1-a F, \\
& g_{4}=2 y_{Y}^{2}+2 z_{Y}^{2}-a^{2} x_{Y}, \\
& g_{5}=y_{Y}^{2}+z_{Y}^{2}+\left(b^{2}-1\right) x_{Y}^{2}+b y_{Y} z_{Y}-\epsilon_{X} \epsilon_{Y} y_{Y}+\left(a+2\left(1-\epsilon_{Y}\right)\right) x_{Y}-a F-\left(1-\epsilon_{Y}\right)^{2}, \\
& g_{6}=y_{Y}^{2}+z_{Y}^{2}-x_{Y}^{2}+b y_{Y} z_{Y}+\left(b^{2}+a+2\right) x_{Y}-1-a F,
\end{aligned}
$$

while the $h_{k}$ 's may also be calculated but will not be useful in our analysis.

In Figs. 6-8, we plot the mode frequencies $g_{k}$ over a sample time interval for various parameter combinations corresponding to chaos (Fig. 6), hyperchaos (Fig. 7), and periodic orbits (Fig. 8). When two or more mode frequencies are positive and equal or nearly equal, then we are in the regime where the modes are competitive. We find that both the chaotic and hyperchaotic trajectories give intermittently competitive mode frequencies. That is to say, the more frequencies are competitive frequently (likely a countably infinite number of times) yet not periodic. On the other hand, from Fig. 8 we see that the mode frequencies can become competitive in the case of periodic orbits, yet for those cases the mode frequencies are themselves completely periodic, which rules out chaos. 


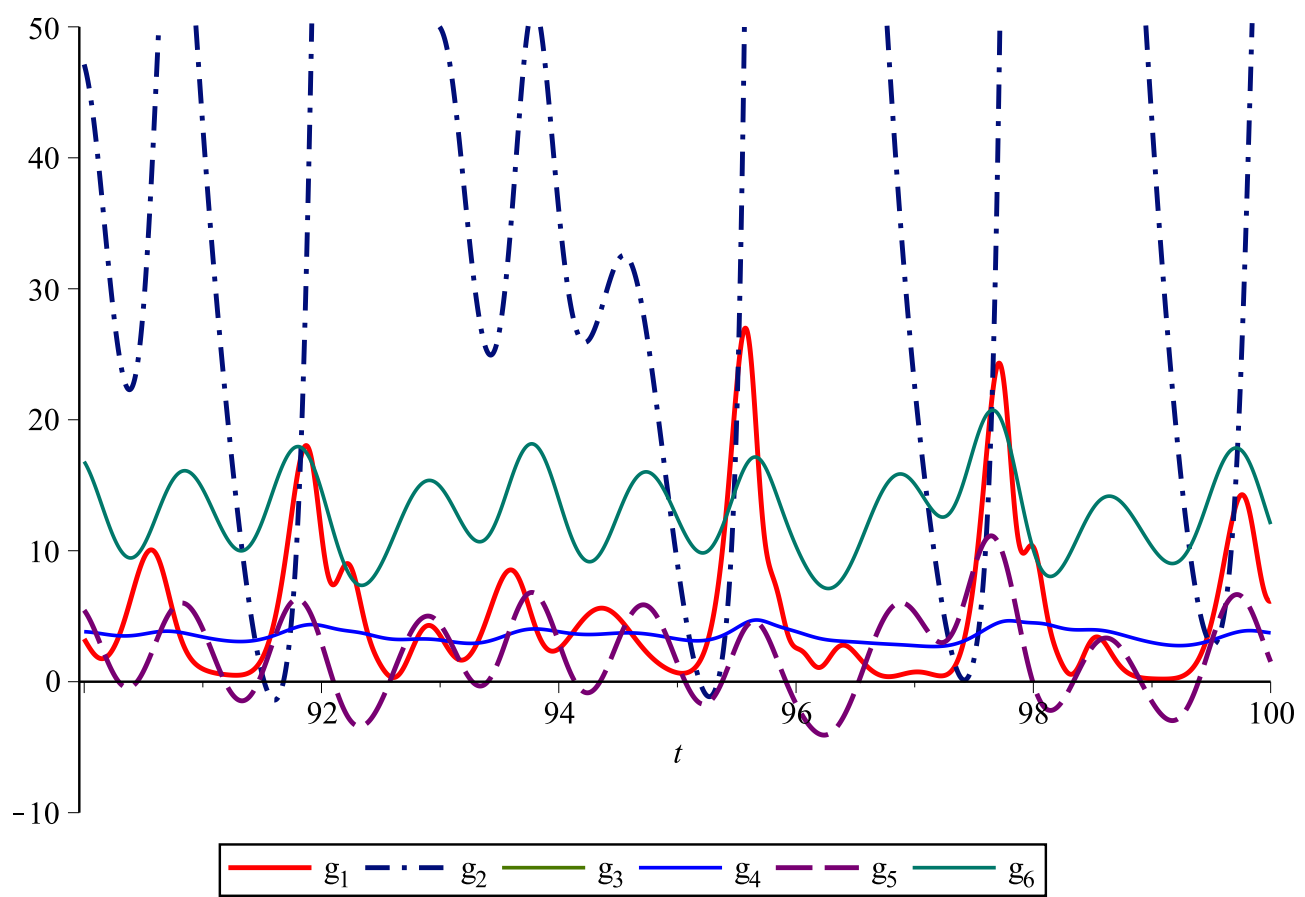

Fig. 7. Plot of the mode frequencies for the parameter values $a=0.25, b=4, F=8, G_{Y}=0.1, G_{X}=0.5, \epsilon_{X}=4, \epsilon_{Y}=0.25$ giving hyperchaos.

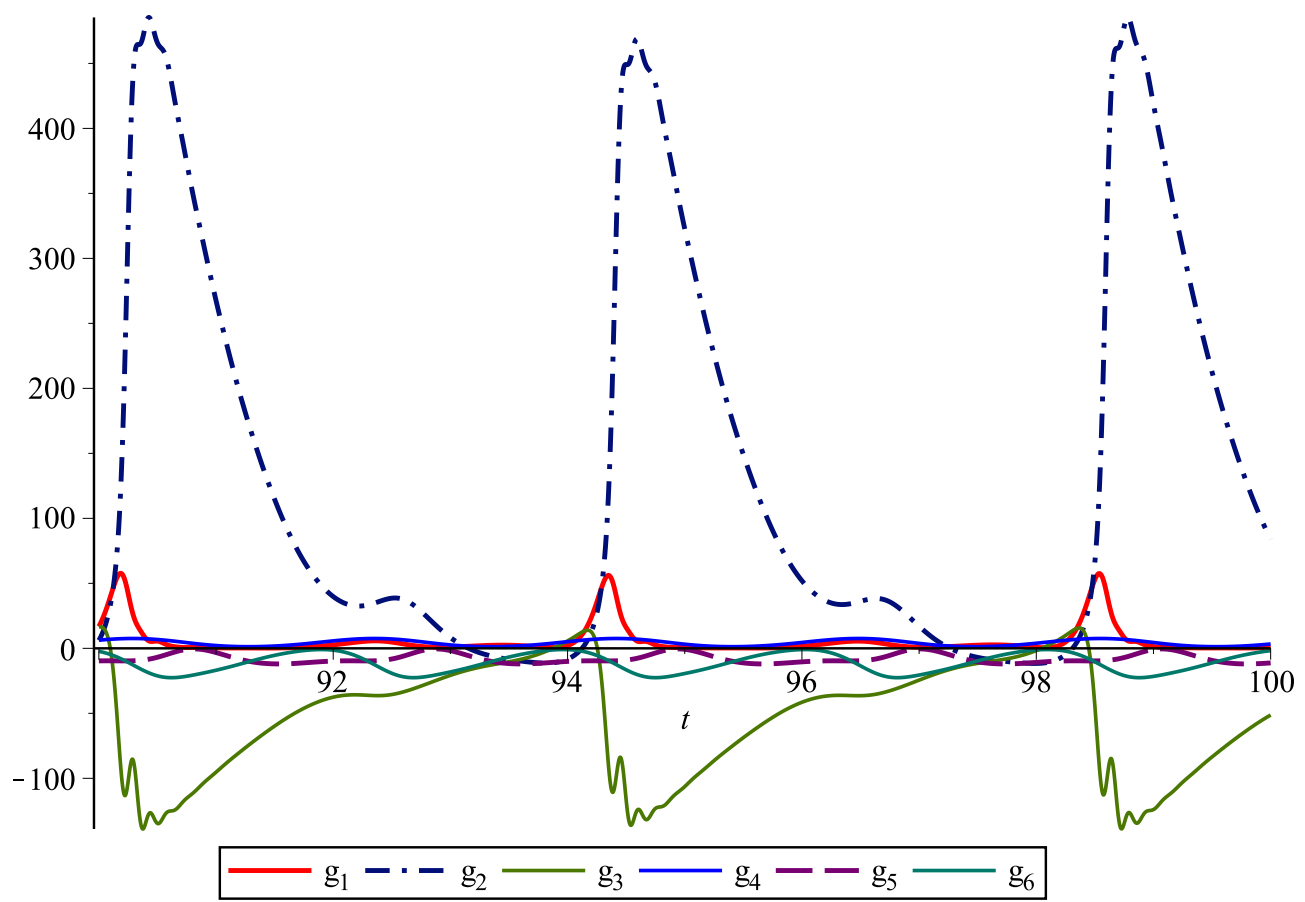

Fig. 8. Plot of the mode frequencies for the parameter values $a=0.25, b=4, F=8, G_{Y}=3, G_{X}=0.2, \epsilon_{X}=4$, $\epsilon_{Y}=0$ giving periodic solutions. Note that the more are more often negative, most intersections of the mode frequency curves occur when they take negative values, and the interaction that do take place between $g_{1}$ and $g_{2}$ are periodic. As mentioned elsewhere, one should look for intermittency, rather than periodicity, when attempting to use competitive modes to obtain chaotic trajectories. 
While competitive modes can be used in order to search for chaos, yet further numerical simulation should be used to verify any chaos predicted. In this way, we can view the competitive modes analysis as a diagnostic tool useful when searching for chaos, although the existence or two or more competitive modes should be viewed more as a necessary condition for chaos, rather than sufficient condition. This reinforces the point of view discussed in [Van Gorder, 2016].

\section{Conclusions}

We proposed a coupled Lorenz-84 system which serves as a crude model of stratosphere-troposphere interactions. We included symmetric and asymmetric forcing parameters for each layer of the atmosphere, as well as parameters $\epsilon_{X}$ (coupling stratospheric dynamics to the troposphere) and $\epsilon_{Y}$ (coupling tropospheric dynamics to the stratosphere). Treating the asymmetric forcing parameters and the coupling parameters as bifurcation parameters, we find that regular periodic dynamics, chaos, and hyperchaos are all possible from the model. In some parameter regimes, the asymmetric forcing is destabilizing, bringing about hyperchaos, while for other parameter regimes the asymmetric forcing induces a stabilizing effect. Furthermore, we find that the additional coupling of the troposphere back into the stratosphere via $\epsilon_{Y}$ can either stabilize or destabilize the dynamics of (3), depending on the parameter regime we consider. For some parameter regimes, we find that the system can transition directly between periodic orbits and hyperchaos, bypassing an intermediate chaos regime. This may happen when both troposphere and stratosphere systems undergo a bifurcation from periodic orbits to chaos in a coincident manner, yet there is no destructive interference between the two systems.

Let us remark that we know of no other coupled stratosphere - troposphere model giving hyperchaos, although we feel that such dynamics should naturally be observed. Indeed, it was rather simple to identify parameter regimes for which hyperchaos exists, and these parameter regimes are not inconsistent with physically reasonable parameter values. This also makes physical sense: Under small forcing and coupling perturbations, the stratosphere portion of model (4) can be driven chaotic. Meanwhile, it is already wellknown that the Lorenz-84 model gives chaos, and this is exactly the troposphere component of the model. Therefore, it is reasonable to find hyperchaos in a model where both troposphere and stratosphere dynamics are mutually coupled. Physically, these dynamics are supported in part by the experimental observation of strong turbulence around the tropopause where both layers meet [Shapiro, 1980]. This turbulence has been suggested as one mechanism allowing chemical transport between both layers [Haynes et al., 1995; Shapiro, 1980]. In our model, turbulence occurring around the tropopause would manifest as hyperchaos, while regular chaos only in the tropospheric part of the model would be turbulence strictly below the tropopause. Therefore, the hyperchaos here has a clear physical relevance, in the context of this previously observed atmospheric turbulence around the tropopause. Of course, one could consider adding terms or additional equations to the model (3) in future work. However, we feel that the rather simple model is still useful in that it detects this tropopause turbulence under a relatively simple framework.

\section{References}

Adusumilli, S. [2015] Stratosphere - Troposphere Interactions Diagnosed through Complex Network Analysis. MSci Dissertation, University of Oxford.

Ali, M.K. \& Fang, J.-Q. [1997] "Synchronization of chaos and hyperchaos using linear and nonlinear feedback functions," Phys. Rev. E 55, 5285.

Baier, G. \& Thomsen, J.S. [1993] "Prototypes of attractors in four dimensions," Phys. Rev. E 48, R4172(R).

Baier, G. \& Sahle, S. [1995] "Design of hyperchaotic flows," Phys. Rev. E 51, R2712(R).

Bryant, P.H. [2010] "Optimized synchronization of chaotic and hyperchaotic systems," Phys. Rev. E 82, 015201(R).

Choudhury, S. R. \& Van Gorder, R. A. [2012] "Competitive modes as reliable predictors of chaos versus hyperchaos and as geometric mappings accurately delimiting attractors," Nonlinear Dynamics 69(4), 2255-2267.

Colet, P., Roy, R. \& Wiesenfeld, K. [1994] "Controlling hyperchaos in a multimode laser model," Phys. Rev. E 50, 3453. 
Dee, D.P. et al. [2011] "The ERAInterim reanalysis: Configuration and performance of the data assimilation system," Quarterly Journal of the Royal Meteorological Society 137(656), 553.

Gao, T., Chen, Z., Yuan, Z. \& Chen, G. [2006] "A hyperchaos generated from Chen's system," International Journal of Modern Physics C 17, 471.

Grassi, G, Severance, F. L. \& Miller, D. A. [2009] "Multi-wing hyperchaotic attractors from coupled Lorenz systems," Chaos, Solitons \& Fractals 41, 284-291.

Haynes, P.H., Mcintyre, M.E., Douglass, A.R., Rood, R.B. \& Pfister, L. [1995] "Stratosphere-troposphere exchange," Rev. Geophys 33(4), 403.

Huang, D. [2004] "Stabilizing near-nonhyperbolic chaotic systems with applications," Phys. Rev. Lett. 93, 214101.

Kapitaniak, T. [1994] "Synchronization of chaos using continuous control," Phys. Rev. E 50, 1642.

Kapitaniak, T., Maistrenko, Y. \& Popovych, S. [2000] "Chaos-hyperchaos transition," Phys. Rev. E 62, 1972.

Kapitaniak, T. [1993] "Transition to hyperchaos in chaotically forced coupled oscillators," Phys. Rev. E 47, R2975(R).

Kestler, J., Kinzel, W. \& Kanter, I. [2007] "Sublattice synchronization of chaotic networks with delayed couplings," Phys. Rev. E 76, 035202(R).

Kistler, R. et al. [2001] "The NCEP-NCAR 50 year reanalysis: Monthly means CD-ROM and Documentation," Bulletin of the American Meteorological Society 82(2), 247.

Lorenz, E.N. [1963] "Deterministic nonperiodic flow," Journal of the Atmospheric Sciences 20(2), 130.

Lorenz, E.N. [1984] "Irregularity: a fundamental property of the atmosphere," Tellus A 36(2), 98.

Lorenz, E.N. [1990] "Can chaos and intransitivity lead to interannual variability?," Tellus A 42(3), 378.

Ma, S.-C., Chen, C.-C. \& Chen, B.-W. [1997] "Dynamics and transitions of the coupled Lorenz system," Phys. Rev. E 56, 1550.

Macek, W.M. \& Strumik, M. [2014] "Hyperchaotic intermittent convection in a magnetized viscous fluid," Phys. Rev. Lett. 112, 074502.

Matthewman, N.J., Esler, J.G., Charlton-Perez, A.J. \& Polvani, L.M. [2009] "A new look at stratospheric sudden warmings. Part III: Polar vortex evolution and vertical structure," Journal of Climate 22(6), 1566.

McCullen, N.J. \& Moresco, P. [2011] "Route to hyperchaos in a system of coupled oscillators with multistability," Phys. Rev. E 83, 046212.

Moser, H.R., Meier, P.F. \& Waldner, F. [1993] "Numerical simulations of chaotic and hyperchaotic signals measured in nonlinear magnetic resonance," Phys. Rev. B 47, 217.

Nik, H.S. \& Van Gorder, R.A. [2013] "Competitive modes for the Baier-Sahle hyperchaotic flow in arbitrary dimensions," Nonlinear Dynamics 74(3), 518.

Pavlov, A.N., Pavlova, O.N., Mohammad, Y.K. \& Kurths, J. [2015] "Characterization of the chaoshyperchaos transition based on return times," Phys. Rev. E 91, 022921.

Peinke, J., Richter, R., Parisi, J. [1993] "Spatial coherence of nonlinear dynamics in a semiconductor experiment," Phys. Rev. B 47, 115.

Peng, J.H., Ding, E.J., Ding, M. \& Yang, W. [1996] "Synchronizing hyperchaos with a scalar transmitted signal," Phys. Rev. Lett. 76, 904.

Reeves, B., Van Gorder, R. A. \& Choudhury, S. R. [2012] "Chaotic regimes, post-bifurcation dynamics, and competitive modes for a generalized double Hopf normal form," Int. J. of Bifurcation and Chaos 22, 1250292.

Rössler, O.E. [1979] "An equation for hyperchaos," Physics Letters A 71, 155.

Shapiro, M.A. [1980] "Turbulent mixing within tropopause folds as a mechanism for the exchange of chemical constituents between the stratosphere and troposphere," Journal of the Atmospheric Sciences 37(5), 994.

Shepherd, T.G. [2002] "Issues in stratosphere-troposphere coupling," Journal of the Meteorological Society of Japan 80(4B), 769.

Shuai, J.W., Chen, Z.X., Liu, R.T. \& Wu, B.X. [1997] "Maximum hyperchaos in chaotic nonmonotonic neuronal networks," Phys. Rev. E 56, 890. 
Solomon, S. [2007] Climate change 2007-the physical science basis: Working group I contribution to the fourth assessment report of the IPCC, volume 4. Cambridge University Press.

Tamasevicius, A. \& Cenys, A. [1997] "Synchronizing hyperchaos with a single variable," Phys. Rev. E 55, 297.

Thompson, D.W.J., Wallace, D.M. \& Hegerl, G.C. [2000] "Annular modes in the extratropical circulation. Part II: Trends," Journal of Climate 13(5), 1000.

Udaltsov, V.S., Goedgebuer, J.-P., Larger, L. \& Rhodes, W.T. [2001] "Communicating with optical hyperchaos: Information encryption and decryption in delayed nonlinear feedback systems," Phys. Rev. Lett. 86, 1892.

Van Gorder, R. A. [2011] "Emergence of chaotic regimes in the generalized Lorenz canonical form: A competitive modes analysis," Nonlin. Dyn. 66, 153-160.

Van Gorder, R. A. [2013] "Triple mode alignment in a canonical model of the the blue-sky catastrophe," Non. Dyn. 73, 397-403.

Van Gorder, R. A. [2016] "A third-order extension to the Linard oscillator and its competitive modes analysis," Non. Dyn. in press, doi:10.1007/s11071-016-2885-z

Van Gorder, R. A. \& Choudhury, S. R. [2010] "Classification of chaotic regimes in the T system by use of competitive modes," Int. J. Bifurcation and Chaos 20, 3785-3793.

Wolf, A., Swift, J.B., Swinney, H.L. \& Vastano, J.A. [1985] "Determining Lyapunov exponents from a time series," Physica D 16(3), 285.

Xiao, J.H., Hu, G. \& Qu, Z. [1996] "Synchronization of spatiotemporal chaos and its application to multichannel spread-spectrum communication," Phys. Rev. Lett. 77, 4162.

Yao, W., Yu, P. \& Essex, C. [2002] "Estimation of chaotic parameter regimes via generalized competitive mode approach," Commun. Nonlin. Sci. Numer. Simul. 7, 197-205.

$\mathrm{Yu}, \mathrm{P}$. [2006] "Bifurcation, limit cycles and chaos of nonlinear dynamical systems," Bifurcation and Chaos in Complex Systems, eds. Sun, J.-Q. \& Luo, A. C. J.

Yu, P., Yao, W. \& Chen, G. [2007] "Analysis on topological properties of the Lorenz and the Chen attractors using GCM," Int. J. Bifurcation and Chaos 17, 2791-2796. 